\title{
OEIS Complex (Omphalocele, Exstrophy of Bladder, Imperforate Anus and Spine Defects)
}

\section{Kandavadivelu $\mathbf{M}^{*}$ \\ PSG Institute of Medical Sciences \& Research, India}

*Corresponding author: Manickavasuki Kandavadivelu, PSG Institute of Medical

Sciences \& Research, India, Tel: 9842766782; E-mail: vasukikalyan01@gmail.com

\section{Mini Review}

Volume 2 Issue 1

Received Date: January 22, 2018

Published Date: February 16, 2018

\begin{abstract}
OEIS complex comprises of a combination of defects including Omphalocele, Exstrophy of bladder, Imperforate anus and Spinal defects. The prenatal ultrasound of the fetus may show grossly malformed fetus. It may be provisionally diagnosed as OEIS complex by Non - visualization of urinary bladder, presence of Omphalocele, limb and spine defects in the ultrasound. During dissection of the fetus short umbilical cord and adherent amniotic membrane may be observed. On opening the sac, liver, intestines and cloaca were found to be contents. External genitalia and anal opening may not be seen. There may be an imperforate anus. Fetal spine may shows scoliosis, hemivertebrae or any other vertebral anomalies.

Prognosis of the fetus depends on the associated anomalies and its severity. Serial ultrasonography may be used to accurately diagnose abdominal wall defect in utero and to diagnose the associated anomalies. The possibility of correction of the defects after birth of the fetus or termination of the fetus may be determined by the Ultrasound.
\end{abstract}

Keywords: Exstrophy of bladder; Omphalocele; Scoliosis; Umbilical cord

\section{Introduction}

OEIS complex comprises of a combination of defects including Omphalocele, Exstrophy of bladder, Imperforate anus and Spine defects. It results from improper closure of ventral abdominal wall due to failure of convergence of cephalo - caudal and lateral folding's of embryo during $4^{\text {th }}$ week of development and is associated with defects in development of cloaca and urorectal septum. It occurs in 1 in 2,00,000 to 4,00,000 pregnancies and is of unknown cause.

Omphalocele is an embryological defect of the umbilical ring and medial segments of the two lateral abdominal folds during fetal growth. This is a persistence of the herniation of the abdominal contents into the proximal part of the umbilical cord. Herniation of the intestines into the cord occurs in approximately 1 in 5000 births and herniation of liver and intestines occur in 1 in approximately 10,000 births. The abdominal cavity is small. Omphalocele results from impaired growth of four components of the abdominal wall. Because formation of abdominal compartment occurs during gastrulation, a critical failure of growth at this time is often associated with other congenital anomalies involving cardiac and urogenital systems [1]. It represents when portions of the midgut fails to return to abdominal cavity from its physiological umbilical herniation during $6^{\text {th }}$ to $10^{\text {th }}$ weeks. This can be divided into two groups depending on the size of the hernia defect. A major or giant omphalocele 
is classified as $5 \mathrm{~cm}$ or large defect and minor omphalocele occurs when the defect is $4 \mathrm{~cm}$ or less. The hernia sac may contain small and large bowel, stomach, liver, spleen, urinary bladder, gonads and rarely heart and lungs. The defect is covered by amnion as umbilical cord and is covered by its reflections.

Bladder Exstrophy (Ectopia vesicae) is a congenital anomaly that exists along the spectrum of Exstrophy Epispadias complex. It is often associated with Epispadias. It involves protrusion of urinary bladder through a defect in the abdominal wall. It may be due to abnormalities of bony pelvis, pelvic floor and genitalia. It is due to failed reinforcement of the cloacal membrane by underlying mesoderm.

An imperforate anus or anorectal malformations (ARMs) are birth defects in which the rectum is malformed.

There are several forms of imperforate anus and anorectal malformations. The classical Wingspread classification was in low and high anomalies:

- A low lesion, in which the colon remains close to the skin. In this case, there may be a stenosis (narrowing) of the anus, or the anus may be missing altogether, with the rectum ending in a blind pouch.

- A high lesion, in which the colon is higher up in the pelvis and there is a fistula connecting the rectum and the bladder, urethra or the vagina.

- A persistent cloaca, in which the rectum, vagina and urinary tract are joined into a single channel.

Imperforate anus is usually present along with other birth defects-spinal problems, heart problems, tracheoesophageal fistula, esophageal atresia, renal anomalies, and limb anomalies are among the possibilities.

Commonest entities associated with Imperforate anus are VACTERL (Vertebral anomalies, Anal atresia, Cardiovascular anomalies, Tracheo oesophageal fistula, Esophageal atresia, Renal and Limb anomalies) syndrome, Urorectal septum malformation sequence and OEIS complex.

Prognosis of OEIS Complex depends on the associated anomalies and its severity. Serial ultrasonography may be used to accurately diagnose abdominal wall defect in utero for monitoring the fetal growth.

\section{Discussion}

The first description of OEIS complex was published by Littre, et al. in 1709 [2]. The term OEIS complex was first used by Carey et al in1978. He described a congenital syndrome with multiple malformations including Omphalocele, Exstrophy of bladder, imperforate anus and spine defects [3]. Previous terminologies were Ectopia of cloaca, Vesico - intestinal fissure, Exstrophy splanchnia. Subsequently in addition to four clinical manifestations, its association with genital, renal and skeletal abnormalities like Spina bifida, Symphysis pubis diastasis and limb abnormalities were also demonstrated [4].

Etiology is unknown but several associations have been suggested. Most frequently it is of sporadic in occurrence. Possibility of teratogenic exposure with Diazepam, Diphenylhydantoin and genetic factors such as Trisomy 18 [5]. Single gene defects in Blastogenesis and mutations in Homeobox genes such as HLXB9 have been suggested. Higher incidence in monozygotic twins suggests a possible genetic contribution to occurrence of this multisystem defect [6].

The surgical treatment of OEIS complex can be achieved in a single or multiple stages with increasing preference towards the staged approach. The goals of OEIS management are: separating the bowel from the hemi-bladders to create an intestinal stoma, closing the omphalocele, adaptation of bladder halves and adequate cosmetic and functional urogenital reconstructions with preserving the renal functions.

\section{Conclusion}

OEIS complex is a rare congenital anomaly with unknown etiology and varied presentation with most of the case are diagnosed on autopsy. Chromosomal abnormality, though rare should be sought for whenever possible. OEIS complex must be ruled out in case of Omphalocele and non - visualization of bladder on antenatal ultrasound. 3D - ultrasound, Doppler, fetal MR are complementary techniques that may aid the prenatal diagnosis or detection of congenital disease.

Nowadays OEIS complex if diagnosed, the termination of a fetus is not an ideal one. Serial ultrasound can be done to accurately diagnose abdominal wall defect in utero for monitoring the fetal growth. Most of the OEIS complex fetuses survive with advances in perinatal caring and surgical reconstruction procedures.

Management varies from interruption of the pregnancy in severe cases to appropriate perinatal management in less severe cases by team of obstetricians, neonatologists, pediatric surgeons and neurosurgeons.

Staged reconstruction is an appropriate approach. 
We have to allow the OEIS complex - fetus up to the period of full term and to deliver even though if it is diagnosed early (without termination), because staged surgical reconstruction and survival of the fetus are possible factors which help in the survival of the fetus.

\section{References}

1. Keith LM, Persaud TVN (2008) The Developing human, Clinically oriented Embryology. $8^{\text {th }}$ (Edn.), 11: 228-229.

2. Smith NM, Chambers HM, Furness ME, Haan EA (1992) The OEIS complex: recurrence in siblings. J Med Genet 29: 730-32.
3. Shanske AL, Pande S, Aref K, Vega R, Brion L, et al. (2003) Omphalocele - Exstrophy - imperforate anusspinal defects (OEIS) in triplet pregnancy after IVF \&CVS. Birth defects res 67(6): 467-471.

4. Kallen K, Castilla EE, Robert E, Mastrioacovo P, Kallen B (2000) OEIS complex - a population study. Am J Med Genet 92(1): 62-68.

5. Keppler-Noreiul KM (2001) OEIS: a review of 14 cases. Am J Med Genet 99(4): 271-279.

6. Lee DH, Cottrell JR, Sander RC, Meyers CM, Wulfsberg CA, et al. (1994) OEIS complex in monozygotic twins. Am J Med Genet 84(1): 29-33. 